\title{
THE EULER NUMBER OF THE NORMALIZATION OF AN ALGEBRAIC THREEFOLD WITH ORDINARY SINGULARITIES
}

\author{
SHOJI TSUBOI \\ Department of Mathematics and Computer Science \\ Kagoshima University \\ Kourimoto 1-21-35, 890-0065 Kagoshima, Japan \\ E-mail: tsuboi@sci.kagoshima-u.ac.jp
}

\begin{abstract}
By a classical formula due to Enriques, the Euler number $\chi(X)$ of the nonsingular normalization $X$ of an algebraic surface $S$ with ordinary singularities in $P^{3}(\mathbf{C})$ is given by $\chi(X)=n\left(n^{2}-4 n+6\right)-(3 n-8) m+3 t-2 \gamma$, where $n$ is the degree of $S, m$ the degree of the double curve (singular locus) $D_{S}$ of $S, t$ is the cardinal number of the triple points of $S$, and $\gamma$ the cardinal number of the cuspidal points of $S$. In this article we shall give a similar formula for an algebraic threefold with ordinary singularities in $P^{4}(\mathbf{C})$ which is free from quadruple points (Theorem 4.1).
\end{abstract}

1. Preliminaries. We begin with recalling some definitions.

Definition 1 ([1]). An irreducible hypersurface $S$ in the complex projective 3 -space $P^{3}(\mathbf{C})$ is called an algebraic surface with ordinary singularities if it is locally isomorphic to one of the following germs of hypersurface at the origin of the complex 3 -space $\mathbf{C}^{3}$ at every point of $S$ :
(i) $z=0$
(simple point)
(ii) $y z=0$
(ordinary double point)
(iii) $x y z=0$
(ordinary triple point)
(iv) $x y^{2}-z^{2}=0$ (cuspidal point),

where $(x, y, z)$ is the coordinate on $\mathbf{C}^{3}$.

2000 Mathematics Subject Classification: Primary 32S20; Secondary 32S25, 14C17, 14C21.

Key words and phrases: Euler number, threefold, ordinary singularities.

This work is partly supported by the Grand-in-Aid for Scientific Research (No. 13640083), The Ministry of Education, Science and Culture, Japan.

The paper is in final form and no version of it will be published elsewhere. 
Definition 2 ([6]). An irreducible hypersurface $T$ in the complex projective 4-space $P^{4}(\mathbf{C})$ is called an algebraic threehold with ordinary singularities if it is locally isomorphic to one of the following germs of hypersurface at the origin of the complex 4 -space $\mathbf{C}^{4}$ at every point of $T$ :
(i) $w=0$
(simple point)
(ii) $z w=0$
(ordinary double point)
(iii) $y z w=0$
(ordinary triple point)
(iv) $x y z w=0$
(ordinary quadruple point)
(v) $x y^{2}-z^{2}=0 \quad$ (cuspidal point)
(vi) $w\left(x y^{2}-z^{2}\right)=0$ (stationary point),

where $(x, y, z, w)$ is the coordinate on $\mathbf{C}^{4}$.

It is known that every complex projective surface (resp. threefold) is birationally equivalent to an algebraic surface (resp. threefold) with ordinary singularities.

Next we give the definition of the polar classes of an $r$-dimensional subvariety $X^{r}$ in a complex projective space $P^{n}(\mathbf{C})$. Denote by $U$ the open subset of $X^{r}$ consisting of all simple points of $X$. For a given linear $(n-r+k-2)$-dimensional subspace $L_{(k)}$ of $P^{n}(\mathbf{C})$, we let $M_{k}(U)$ denote the locus of points $x \in U$ such that the tangent space $T_{x} X$ of $X$ at $x$ intersects $L_{(k)}$ in a space at least $k-1$ dimension.

Definition 3. The closure $M_{k}$ of $M_{k}(U)$ in $X$ is called the $k$-th polar locus of $X$.

$M_{k}$ has a natural reduced scheme structure and, for a general $L_{(k)}, M_{k}$ has codimension $k$ in $X$. Moreover, for such $L_{(k)}$, the rational equivalent class of the cycle defined by $M_{k}$ does not depend on $L_{(k)}\left(\mathrm{cf}\right.$. [5]). This class is denoted by $\left[M_{k}\right]$.

Definition 4 . The class $\left[M_{k}\right]$ is called the $k$-th polar class of $X$. The degree $\mu_{k}$ of $M_{k}$ is called the $k$-th class. The top class $\mu_{r}$ is called the class of $X$.

Now we give the definition of the Segre class of a closed subscheme $X$ of a scheme $Y$. We denote by $\mathcal{I}$ the ideal sheaf of $X$ in $Y$ and put

$$
S^{\cdot}:=\sum_{k=0}^{\infty} \mathcal{I}^{k} / \mathcal{I}^{k+1},
$$

which is a graded sheaf of $\mathcal{O}_{X}$-algebras on $X$. To $S^{\cdot}$ we associate two schemes over $X$ : the cone of $S^{\cdot}$

$$
C:=\operatorname{Spec}\left(S^{\cdot}\right), \quad \pi: C \rightarrow X
$$

and the projective cone $P(C)$ to $X$ in $Y$ by

$$
P(C):=\operatorname{Proj}\left(S^{\cdot}\right), \quad p: P(C) \rightarrow X .
$$

$C$ is called the normal cone to $X$ in $Y$, denoted by $C_{X} Y$, and $P(C)$ the projective normal cone to $X$ in $Y$. On $P(C)$ there is a canonical line bundle, denoted by $\mathcal{O}_{C}(1)$. Let $z$ be a variable, $S^{\cdot}[z]$ the graded algebra whose $n$-th graded piece $\left(S^{\cdot}[z]\right)^{n}$ is

$$
S^{n} \oplus S^{n-1} z \oplus \ldots \oplus S^{1} z^{n-1} \oplus S^{0} z^{n}
$$


The corresponding cone is denoted by $C \oplus 1$. The cone

$$
P(C \oplus 1)=\operatorname{Proj}\left(S^{\cdot}[z]\right), \quad q: P(C \oplus 1) \rightarrow X
$$

is called the projective completion of $C$. The element $z$ in $\left(S^{\cdot}[z]\right)^{1}$ determines a regular section of $\mathcal{O}_{C \oplus 1}(1)$ on $P(C \oplus 1)$ whose zero-scheme is canonically isomorphic to $P(C)$. The complement to $P(C)$ in $P(C \oplus 1)$ is canonically isomorphic to $C$.

Definition 5. The Segre class of $X$ in $Y$, denoted by $s(X, Y)$, is the class in the graded Chow group $A_{*} X$ of $X$ defined by the formula

$$
s(X, Y):=q_{*}\left(\sum_{i \geq 0} c_{1}\left(\mathcal{O}_{C \oplus 1}(1)\right)^{i} \cap[P(C \oplus 1)]\right) .
$$

Note that $s(X, Y)$ is a birational invariant, which means that if $f: Y^{\prime} \rightarrow Y$ is a morphism of pure-dimensional schemes, $X \subset Y$ a closed subscheme, $X^{\prime}=f^{-1}(X)$ the inverse image scheme, then the Segre class of $X^{\prime}$ in $Y^{\prime}$ pushes forward to the Segre class of $X$ in $Y$. If the normal cone $C_{X} Y$ is a vector bundle $N$, then $s(X, Y)=c(N)^{-1} \cap[X]$ where $c(N)^{-1}$ denotes the total inverse Chern class of $N$ (cf. [2], Chapter 4).

Finally, we give the definitions of regular embeddings and local complete intersection morphisms of schemes.

Definition 6. We say a closed embedding $\iota: X \rightarrow Y$ of schemes is a regular embedding of codimension $d$ if every point in $X$ has an affine neighborhood $U$ in $Y$ such that if $A$ is the coordinate ring of $U, I$ the ideal of $A$ defining $X$, then $I$ is generated by a regular sequence of length $d$.

If this is the case, the conormal sheaf $\mathcal{I} / \mathcal{I}^{2}$, where $\mathcal{I}$ is the ideal sheaf of $X$ in $Y$, is a locally free sheaf of rank $d$. The normal bundle to $X$ in $Y$, denoted by $N_{X} Y$, is the vector bundle on $X$ whose sheaf of sections is dual to $\mathcal{I} / \mathcal{I}^{2}$. Note that the normal bundle $N_{X} Y$ is canonically isomorphic to the normal cone $C_{X} Y$ for a (closed) regular embedding $\iota: X \rightarrow Y$ since the canonical map from $\operatorname{Sym}\left(\mathcal{I} / \mathcal{I}^{2}\right)$ to $S:=\sum_{k=0}^{\infty} \mathcal{I}^{k} / \mathcal{I}^{k+1}$ is an isomorphism (cf. [2], Appendix B, B.7).

Definition 7. A morphism $f: X \rightarrow Y$ is called a local complete intersection morphism of codimension $d$ if $f$ factors into a (closed) regular embedding $\iota: X \rightarrow Y$ of some constant codimension $e$, followed by a smooth morphism $p: P \rightarrow Y$ of constant relative dimension $d+e$.

2. The existence of a good linear pencil of hyperplane sections. Throughout this section we denote by $X$ an algebraic threefold with ordinary singularities of degree $n$ in the complex projective 4-space $P^{4}(\mathbf{C})$, by $D$ the double surface of $X$, i.e., the singular locus of $X$, by $T$ the triple points locus of $X$, by $C$ the cuspidal point locus of $X$, by $\sum s$ the stationary point locus of $X$. Let $m, t, \gamma$ be the degrees of $D, T, C$, respectively. Let $P_{\infty}$ be a two-dimensional linear subspace of $P^{4}(\mathbf{C})$ such that $C_{\infty}:=P_{\infty} \cap X$ is an irreducible curve with ordinary double points in $P_{\infty} \simeq P^{2}(\mathbf{C})$. Let $P$ be a one-dimensional linear subspace of $P^{4}(\mathbf{C})$ situated in twisted position with respect to $P_{\infty}$, i.e., the linear subspace $L\left(P_{\infty}, P\right)$ generated by $P_{\infty}$ and $P$ is equal to $P^{4}(\mathbf{C})$. Let $\pi: X \backslash C_{\infty} \rightarrow P$ be the linear projection with center $C_{\infty}$, i.e., $\pi(x):=H_{x} \cap P$ for $x \in X \backslash C_{\infty}$, where 
$H_{x}=L\left(x, P_{\infty}\right)$ is the hyperplane generated by $x$ and $P_{\infty}$. We put $X_{\lambda}:=H_{\lambda} \cap X$ for $\lambda \in P$ and $\mathcal{L}:=\bigcup_{\lambda \in P} X_{\lambda}$. Then $\mathcal{L}$ is a linear system on $X$ with the base point locus $B s(\mathcal{L})=C_{\infty}$. Let $f: X_{1} \rightarrow X$ be the normalization map and $\widetilde{\mathcal{L}}:=\bigcup_{\lambda \in P} \widetilde{X_{\lambda}}$ the pull-back of $\mathcal{L}$ to $X_{1}$.

THEOREM 2.1. If we take $P_{\infty}$ sufficiently general, there exists a finite set $\left\{\lambda_{1}, \ldots, \lambda_{c}\right\}$ of points of $P$ such that

(i) $\widetilde{X_{\lambda}}$ is non-singular for $\lambda$ with $\lambda \neq \lambda_{i}(1 \leq i \leq c)$, and

(ii) $\widetilde{X_{\lambda_{i}}}$ is a surface with only one isolated ordinary double point which is contained in $X_{1} \backslash f^{-1}\left(C_{\infty}\right)$ for any $i$ with $1 \leq i \leq c$,

where $c$ is the class of $X$.

Proof. We consider the Gauss map

$$
\Phi: X \rightarrow P^{4}(\mathbf{C})^{\vee}
$$

defined by

$$
\Phi(p)=\left[\frac{\partial F}{\partial x_{0}}(p): \frac{\partial F}{\partial x_{1}}(p): \frac{\partial F}{\partial x_{2}}(p): \frac{\partial F}{\partial x_{3}}(p): \frac{\partial F}{\partial x_{4}}(p)\right]
$$

for $p \in X$, where $F$ is the homogeneous polynomial defining $X$ in $P^{4}(\mathbf{C}),\left[x_{0}: x_{1}: x_{2}\right.$ : $x_{3}: x_{4}$ ] the homogeneous coordinate on $P^{4}(\mathbf{C})$, and $P^{4}(\mathbf{C})^{\vee}$ the dual projective space of $P^{4}(\mathbf{C}) . \Phi$ is a rational map, which is not defined on the singular locus $D$ of $X$. Let $\bar{X}$ be the closure in $X \times P^{4}(\mathbf{C})^{\vee}$ of the graph of $\Phi$. We denote by $\pi_{1}: \bar{X} \rightarrow X$ the morphism induced by the projection to the first factor, and $\pi_{2}: \bar{X} \rightarrow P^{4}(\mathbf{C})^{\vee}$ the one induced by the projection to the second factor. We call $\pi_{1}: \bar{X} \rightarrow X$ the Nash blow-up of $X$. Note that the rational map $\Phi$ can be extended to $\bar{X}$ and $\bar{X}$ is minimal among the varieties with such property. In our case, since $X$ is a hypersurface, $\bar{X}$ coincides with the blow-up of the Jacobian ideal of $X$ ([4], Remark 2, p. 300). We denote by $X^{\vee}$ the image of $\bar{X}$ by $\pi_{2}: \bar{X} \rightarrow P^{4}(\mathbf{C})^{\vee}$, and call it the dual variety of $X$. The dimension of $X^{\vee}$ is not less than 1, nor greater than 3 ([3], Example 15.22, p. 196).

We are now going to define an algebraic subset $B$ in $P^{4}(\mathbf{C})^{\vee}$, whose points correspond to hyperplanes in $P^{4}(\mathbf{C})$ being in bad positions in some sense at their intersecting points with the cuspidal point locus $C$, or stationary point locus $\sum s$ of $X$. Let $p$ be a point of $C$, or $\sum s$. Then there is an open neighborhood $U$ of $p$ and a complex analytic local coordinates $(x, y, z, w)$ with center $p$ such that the defining equation of $X$ is given by one of the following:

$$
\begin{aligned}
x y^{2}-z^{2} & =0 \\
w\left(x y^{2}-z^{2}\right) & =0 .
\end{aligned}
$$

Let $\left(\zeta_{1}, \zeta_{2}, \zeta_{3}, \zeta_{4}\right)$ be a linear affine coordinate with center $p$, and $H$ a hyperplane passing through $p$, defined by the equation

$$
\sum_{i=1}^{4} a_{i} \zeta_{i}=0 \quad\left(a_{i} \in \mathbf{C}, 1 \leq i \leq 4\right) .
$$


We say $H$ is in a bad position at the point $p$, if the coefficients of the equation (2.4) satisfy the following two conditions:

$$
\begin{aligned}
& \sum_{i=1}^{4} a_{i} \frac{\partial \zeta_{i}}{\partial y}(0)=0 \\
& \sum_{i=1}^{4} a_{i} \frac{\partial \zeta_{i}}{\partial w}(0)=0 .
\end{aligned}
$$

We define $B_{p}$ to be the algebraic subset of $P^{4}(\mathbf{C})^{\vee}$ consisting of all points which correspond to hyperplanes in $P^{4}(\mathbf{C})$ passing through $p$ and being in a bad position at $p$ in the sense defined above. We define an algebraic subset $B$ of $P^{4}(\mathbf{C})^{\vee}$ by

$$
B:=\bigcup_{p \in C} B_{p} .
$$

Let us note that the stationary points are included in $C$, and since $\operatorname{dim} B_{p}=1$, the codimension of $B$ is greater than 1 . We choose a line $L^{*}$ in $P^{4}(\mathbf{C})^{\vee}$ which satisfies all of the following conditions:

$$
\begin{aligned}
& L^{*} \cap\left\{X^{\vee} \backslash \Phi\left(X_{\mathrm{sm}}\right)\right\}=\emptyset, \\
& L^{*} \cap\left(X^{\vee}\right)_{\text {sing }}=\emptyset, \\
& L^{*} \cap B=\emptyset, \\
& L^{*} \text { intersects transversely with } \Phi\left(X_{\mathrm{sm}}\right) \backslash\left(X^{\vee}\right)_{\text {sing }},
\end{aligned}
$$

where $X_{\mathrm{sm}}$ denotes $X \backslash D$, the simple point locus of $X$, and $\left(X^{\vee}\right)_{\text {sing }}$ the singular point locus of $X^{\vee}$. This is always possible because all the codimensions of $X^{\vee} \backslash \Phi\left(X_{\mathrm{sm}}\right),\left(X^{\vee}\right)_{\text {sing }}$ and $B$ are greater than 1 in $P^{4}(\mathbf{C})^{\vee}$. Note that the cardinal number of the set $L^{*} \cap$ $\left\{\Phi\left(X_{\mathrm{sm}}\right) \backslash\left(X^{\vee}\right)_{\text {sing }}\right\}$ is nothing but the class of $X$. We denote by $H_{\lambda}$ the hyperplane in $P^{4}(\mathbf{C})$ corresponding to each $\lambda \in L^{*}$. We put $X_{\lambda}:=X \cap H_{\lambda}$ and consider the linear pencil

$$
\mathcal{L}=\bigcup_{\lambda \in L^{*}} X_{\lambda}
$$

of hyperplane sections of $X$. We are now going to show that the assertions (i) and (ii) of the theorem hold for the pull-back $\widetilde{\mathcal{L}}=\bigcup_{\lambda \in L^{*}} \widetilde{X_{\lambda}}$ of $\mathcal{L}$ to the normal model $X_{1}$ of $X$ by the normalization map $f: X_{1} \rightarrow X$.

Assertion (i). Let $\left\{\lambda_{1}, \ldots, \lambda_{c}\right\}$ be all of the distinct points of $L^{*} \cap\left\{\Phi\left(X_{\mathrm{sm}}\right) \backslash\left(X^{\vee}\right)_{\text {sing }}\right\}$, and $\lambda$ a point $L^{*}$ with $\lambda \neq \lambda_{i}(1 \leq i \leq c)$. Then $\lambda \notin X^{\vee}$. This means that $H_{\lambda}$ is not tangent to $X$ at any point of $X_{\mathrm{sm}}$, and not a limit of tangent hyperplanes to $X_{\mathrm{sm}}$. Hence we infer that $\widetilde{X_{\lambda}}$ is non-singular at every point of $X_{1} \backslash f^{-1}(C)$. Therefore what we have to do is to show that $\widetilde{X_{\lambda}}$ is non-singular at $f^{-1}(p)$ for any point $p \in H_{\lambda} \cap C$. In the subsequence we shall show this fact only when $p$ is a stationary point, since the proof for a cuspidal point is more easy. Assume $p$ is a cuspidal point of $X$ and $p \in H_{\lambda}$. We take a complex analytic local coordinate $(x, y, z, w)$ with center $p$ such that the defining equation of $X$ is given by the equation (2.3). We also take a linear affine coordinate $\left(\zeta_{0}, \zeta_{1}, \zeta_{2}, \zeta_{3}\right)$ with center $p$ and assume that the defining equation of $H_{\lambda}$ is given by the 
equation (2.4). We rewrite the equation (2.4) as

$$
A x+B y+C z+D w=0,
$$

where $A, B, C$ and $D$ are complex analytic functions defined in a neighborhood of $p$. $f^{-1}(p)$ consists of two points, say $q_{1}, q_{2}$, where the normalization map $f: X_{1} \rightarrow X$ is given as follows:

$$
\begin{aligned}
& f_{1}:\left(u_{1}, v_{1}, t_{1}\right) \rightarrow\left(u_{1}^{2}, v_{1}, u_{1} v_{1}, t_{1}\right)=(x, y, z, w), \\
& f_{2}:\left(u_{2}, v_{2}, t_{2}\right) \rightarrow\left(u_{2}, v_{2}, t_{2}, 0\right)=(x, y, z, w) .
\end{aligned}
$$

Here $\left(u_{i}, v_{i}, t_{i}\right)(i=1,2)$ is a complex analytic local coordinate with center $q_{i}$. Then the pull-backs of the defining equation of $H_{\lambda}$ in $(2.12)$ by $f_{i}(i=1,2)$ are given by

$$
A_{1}^{*} u_{1}^{2}+B_{1}^{*} v_{1}+C_{1}^{*} u_{1} v_{1}+D_{1}^{*} t_{1}=0,
$$

and

$$
A_{2}^{*} u_{1}+B_{2}^{*} v_{2}+C_{2}^{*} t_{2}=0
$$

where $A_{i}^{*}, B_{i}^{*}, C_{i}^{*}$ and $D_{i}^{*}(i=1,2)$ are the pull-backs of $A, B, C$ and $D$ by the map $f_{i}$. The equations above give the defining equations of $\widetilde{X_{\lambda}}$ at $q_{1}$ and $q_{2}$, respectively. Concerning the equation $(2.13)$, if $B_{1}^{*}(0) \neq 0$ or $D_{1}^{*}(0) \neq 0$, then $\widetilde{X_{\lambda}}$ is non-singular at $q_{1}$. Assume $B_{1}^{*}(0)=D_{1}^{*}(0)=0$ to the contrary, then $B(0)=D(0)=0$. Since

$$
A(0) x+B(0) y+C(0) z+D(0) w=0
$$

is the equation of the embedded tangent space to $H_{\lambda}$ at $p$ in terms of the local coordinate $(x, y, z, w)$, and since $H_{\lambda}$ is defined by the equation 2.4 , we have

$$
\sum_{i=0}^{4} a_{i} \frac{\partial \zeta_{i}}{\partial y}(0)=B(0)=0 \quad \text { and } \quad \sum_{i=0}^{4} a_{i} \frac{\partial \zeta_{i}}{\partial w}(0)=D(0)=0 .
$$

On the other hand, since $\lambda \notin B$, this is because of the condition (2.10), we have

$$
\sum_{i=0}^{4} a_{i} \frac{\partial \zeta_{i}}{\partial y}(0) \neq 0 \quad \text { or } \quad \sum_{i=0}^{4} a_{i} \frac{\partial \zeta_{i}}{\partial w}(0) \neq 0 .
$$

This is a contradiction. Therefore we conclude that $B_{1}^{*}(0) \neq 0$ or $D_{1}^{*}(0) \neq 0$, and so $\widetilde{X_{\lambda}}$ is non-singular at $q_{1}$. Concerning the equation $(2.14)$, if $A_{2}^{*}(0)=B_{2}^{*}(0)=C_{2}^{*}(0)=0$, then $A(0)=B(0)=C(0)=0$. This means the equation of the embedded tangent space to $H_{\lambda}$ at $p$ with respect to the local coordinate $(x, y, z, w)$ is $w=0$, that is, $H_{\lambda}$ is tangent to the hypersurface $w=0$ at $p$. But this is a contradiction, because, since $\lambda \notin X^{\vee}, H_{\lambda}$ is not a limit of tangent hyperplanes to $X$ in $P^{4}(\mathbf{C})$ at simple points of $X$. Therefore we conclude that at least one of $A_{2}^{*}(0), B_{2}^{*}(0)$ and $C_{2}^{*}(0)$ is not zero, and so $\widetilde{X_{\lambda}}$ is non-singular at $q_{2}$.

Assertion (ii). From the same reasoning as in the proof of assertion (i) it follows that $\widetilde{X_{\lambda_{i}}}$ is non-singular at every point of $f^{-1}\left(D_{\lambda_{i}}\right)$ where $D_{\lambda_{i}}=X_{\lambda_{i}} \cap D$. Hence it suffices to show that $X_{\lambda_{i}}$ has only one isolated ordinary double point on $X_{\lambda_{i}} \cap X_{\mathrm{sm}}$. By the manner of choosing the line $L^{*}$ in $P^{4}(\mathbf{C})^{\vee}$, the hyperplane $H_{\lambda_{i}}$ is tangent to $X$ at only one point, say $q$, of $X_{\mathrm{sm}}$. Therefore $X_{\lambda_{i}}$ is non-singular at all but one point $q$ of $X_{\lambda_{i}} \cap X_{\mathrm{sm}}$. To prove that $X_{\lambda_{i}}$ has an isolated ordinary double point at $q$, we assume that the homogeneous coordinate $\left[x_{0}: x_{1}: x_{2}: x_{3}: x_{4}\right]$ of $q$ is $[1: 0: 0: 0: 0]$ and $H_{\lambda_{i}}$ is defined by $x_{4}=0$. 
We put $\zeta_{i}=x_{i} / x_{0}(1 \leq i \leq 4)$, and use this linear affine coordinate $\left(\zeta_{1}, \ldots, \zeta_{4}\right)$ in the subsequent arguments. Then $X$ is defined by $F\left(1, \zeta_{1}, \zeta_{2}, \zeta_{3}, \zeta_{4}\right)=0, q$ is the origin $(0, \ldots, 0)$, and $H_{\lambda_{i}}$ is defined by $\zeta_{4}=0$. Since the tangent hyperplane to $X$ at $q$ is the hyperplane $H_{\lambda_{i}}: \zeta_{4}=0$, we have

$$
\begin{aligned}
& \frac{\partial F}{\partial \zeta_{i}}(1,0, \ldots, 0)=0 \quad(1 \leq i \leq 3) \\
& \frac{\partial F}{\partial \zeta_{4}}(1,0, \ldots, 0) \neq 0
\end{aligned}
$$

Because of (2.16), there is an analytic function $\phi\left(\zeta_{1}, \zeta_{2}, \zeta_{3}\right)$ of the variables $\zeta_{1}, \zeta_{2}, \zeta_{3}$ defined in a neighborhood of the origin, which satisfies the following:

$$
\begin{aligned}
& \phi(0,0,0)=0, \\
& F\left(1, \zeta_{1}, \zeta_{2}, \zeta_{3}, \phi\left(\zeta_{1}, \zeta_{2}, \zeta_{3}\right)\right) \equiv 0 \quad \text { (locally). }
\end{aligned}
$$

This means that the defining equation of $X$ in a neighborhood of $q$ is given by

$$
\zeta_{4}=\phi\left(\zeta_{1}, \zeta_{2}, \zeta_{3}\right)
$$

By the same reasoning as before, we have

$$
\frac{\partial \phi}{\partial \zeta_{i}}(0,0,0)=0 \quad(1 \leq i \leq 3)
$$

Hence $\phi$ is expressed as

$$
\phi=\sum_{1 \leq i, j \leq 3} \frac{\partial^{2} \phi}{\partial \zeta_{i} \partial \zeta_{j}}(0) \zeta_{i} \zeta_{j}+O\left(|\zeta|^{3}\right) .
$$

If we regard $\left(\zeta_{1}, \zeta_{2}, \zeta_{3}\right)$ as a local coordinate on $H_{\lambda_{i}}, X_{\lambda_{i}}$ is defined by $\phi\left(\zeta_{1}, \zeta_{2}, \zeta_{3}\right)=0$ in $H_{\lambda_{i}}$. Therefore, if we prove

$$
\operatorname{det}\left(\frac{\partial^{2} \phi}{\partial \zeta_{i} \partial \zeta_{j}}(0)\right) \neq 0
$$

then we can conclude that, after suitable change of local coordinates, the defining equation of $X_{\lambda_{i}}$ will become

$$
\zeta_{1}^{2}+\zeta_{2}^{2}+\zeta_{3}^{2}=0
$$

in a neighborhood of the origin in $H_{\lambda_{i}}$. This proves that assertion (ii) holds. To prove $(2.22)$, we evaluate the Hessian $\operatorname{det}\left(\partial^{2} F / \partial x_{i} \partial x_{j}\right)$ of the homogeneous polynomial $F$ at $q=[1: 0: 0: 0: 0]$.

First we mention some remarks about $\operatorname{det}\left(\partial^{2} F / \partial x_{i} \partial x_{j}(1,0)\right)$, where and in what follows we write $(1,0)$ instead of $(1,0,0,0,0)$ for short. From the Euler identity

$$
\sum_{i=0}^{4} x_{i} \frac{\partial F}{\partial x_{i}}=n F \quad(n=\operatorname{deg} F)
$$

it follows that

$$
\sum_{j=0}^{4} x_{j} \frac{\partial^{2} F}{\partial x_{i} \partial x_{j}}=(n-1) \frac{\partial F}{\partial x_{i}} \quad(0 \leq i \leq 4)
$$


If $x_{0} \neq 0$, by use of (2.24) and (2.23), we can derive

$$
\operatorname{det}\left(\frac{\partial^{2} F}{\partial x_{i} \partial x_{j}}\right)=\left(\frac{n-1}{x_{0}}\right)^{2}\left|\begin{array}{cccc}
\frac{n}{n-1} F & \frac{\partial F}{\partial x_{1}} & \cdots & \frac{\partial F}{\partial x_{4}} \\
\frac{\partial F}{\partial x_{1}} & \frac{\partial^{2} F}{\partial x_{1}^{2}} & \cdots & \frac{\partial^{2} F}{\partial x_{1} \partial x_{4}} \\
\vdots & \vdots & \ddots & \vdots \\
\frac{\partial F}{\partial x_{4}} & \frac{\partial^{2} F}{\partial x_{4} \partial x_{1}} & \cdots & \frac{\partial^{2} F}{\partial x_{4}^{2}}
\end{array}\right|
$$

Therefore, since $F(1,0)=0$ and $\left(\partial F / \partial x_{i}\right)(1,0)=0(1 \leq i \leq 3)$ (cf. (2.15)), we have

$$
\operatorname{det}\left(\frac{\partial^{2} F}{\partial x_{i} \partial x_{j}}(1,0)\right)=(n-1)^{2}\left(\frac{\partial F}{\partial x_{4}}(1,0)\right)^{2} \operatorname{det}\left(\frac{\partial^{2} F}{\partial x_{i} \partial x_{j}}(1,0)\right)_{1 \leq i, j \leq 3}
$$

Here we need to recall that $\Phi(q)=\lambda$ does not belong to $\left(X^{\vee}\right)_{\text {sing }}$ because of condition (2.9). This means the Gauss map $\Phi$ defined by (2.1) gives a biregular morphism between $X$ and $X^{\vee}$ in a neighborhood of $q$. Therefore the right-hand side of (2.26) is not zero, and so we have

$$
\operatorname{det}\left(\frac{\partial^{2} F}{\partial x_{i} \partial x_{j}}(1,0)\right)_{1 \leq i, j \leq 3} \neq 0
$$

since $\left(\partial F / \partial x_{4}\right)(1,0) \neq 0$ (cf. $\left.(2.16)\right)$. On the other hand, derivating the equation (2.18) twice with respect to the variables $\zeta_{1}, \zeta_{2}, \zeta_{3}$ and substituting 0 for all $\zeta_{i}$, we have

$$
\operatorname{det}\left(\frac{\partial^{2} F}{\partial x_{i} \partial x_{j}}(1,0)\right)_{1 \leq i, j \leq 3}=-\left(\frac{\partial F}{\partial x_{4}}(1,0)\right)^{3} \operatorname{det}\left(\frac{\partial^{2} \phi}{\partial \zeta_{i} \partial \zeta_{j}}(1,0)\right)
$$

Since $\left(\partial F / \partial x_{4}\right)(1,0) \neq 0$, by $(2.28)$ and $(2.27)$ we have

$$
\operatorname{det}\left(\frac{\partial^{2} \phi}{\partial \zeta_{i} \partial \zeta_{j}}(1,0)\right) \neq 0
$$

as required. This completes the proof of the theorem.

In what follows we assume that $P_{\infty}$ is sufficiently general so that Theorem 2.1 holds.

LEMMA 2.2. With the notation from Theorem 2.1, we have the following:

(i) $\widetilde{C_{\infty}}:=f^{-1}\left(C_{\infty}\right)$ is a non-singular curve,

(ii) $\widetilde{\mathcal{L}}:=\bigcup_{\lambda \in P} \widetilde{X_{\lambda}}$ is a linear system on $X_{1}$ with the base point locus $B_{s}(\widetilde{\mathcal{L}})=\widetilde{C_{\infty}}$,

(iii) for $\lambda, \mu \in P$ with $\lambda \neq \mu, \widetilde{X_{\lambda}}$ and $\widetilde{X_{\mu}}$ intersect transversely along $\widetilde{C_{\infty}}$.

Proof. We take an affine coordinate neighborhood $U$ of $P^{4}(\mathbf{C})$ with $U \cap P_{\infty} \neq \emptyset$, and work on this neighborhood. Let $\left(\zeta_{1}, \zeta_{2}, \zeta_{3}, \zeta_{4}\right)$ be a linear affine coordinate on $U$. We may assume that

(a) $P_{\infty} \cap T=\emptyset$ and $P_{\infty} \cap C=\emptyset$,

(2.29) (b) $P_{\infty}$ and $X$ intersect transversely at every non-singular point of $X$, and

(c) $P_{\infty}$ and $D$ intersect transversely.

Let $P_{\infty}=H_{0} \cap H_{1}$ where $H_{0}$ and $H_{1}$ are hyperplanes in $P^{4}(\mathbf{C})$, and let $\varphi_{i}$ be a linear function which defines $H_{i}$ on $U$ for $i=1,2$. Note that the linear system $\widetilde{\mathcal{L}}:=\bigcup_{\lambda \in P} \widetilde{X_{\lambda}}$ is defined by $\alpha f^{*} \varphi_{0}+\beta f^{*} \varphi_{1}(\alpha, \beta \in \mathbf{C})$ where $f^{*} \varphi_{i}(i=1,2)$ denotes the pull-back of $\varphi_{i}$ by the normalization map $f: X_{1} \rightarrow X$. Therefore assertion (ii) is trivial. By the assumption 
(2.29b) the assertions (i) and (iii) also trivially hold at $q=f^{-1}(p)$ for a non-singular point $p$ of $X$, so we will prove that the assertions (i) and (iii) hold at $q \in f^{-1}(p)$ for $p \in D \cap U$. We assume that $\bar{X}$ is defined by $X Y=0$ with respect to some complex analytic local coordinate $(X, Y, Z, W)$ with center $p$, and assume that the normalization map $f$ is given by

$$
(u, v, t) \mapsto(0, u, v, t)=(X, Y, Z, W),
$$

where $(u, v, t)$ is a complex analytic local coordinate with center $q:=f^{-1}(p)$. The Jacobian matrix of $f^{*} \varphi_{0}, f^{*} \varphi_{1}$ with respect to $(u, v, t)$ at $q$ is given as follows:

$$
\begin{aligned}
& \frac{\partial\left(f^{*} \varphi_{0}, f^{*} \varphi_{1}\right)}{\partial(u, v, t)}(q) \\
& =\left(\begin{array}{ccc}
\sum_{i=1}^{4} \frac{\partial \zeta_{i}}{\partial Y}(p) \frac{\partial \varphi_{0}}{\partial \zeta_{i}}(p), & \sum_{i=1}^{4} \frac{\partial \zeta_{i}}{\partial Z}(p) \frac{\partial \varphi_{0}}{\partial \zeta_{i}}(p), & \sum_{i=1}^{4} \frac{\partial \zeta_{i}}{\partial W}(p) \frac{\partial \varphi_{0}}{\partial \zeta_{i}}(p) \\
\sum_{i=1}^{4} \frac{\partial \zeta_{i}}{\partial Y}(p) \frac{\partial \varphi_{1}}{\partial \zeta_{i}}(p), & \sum_{i=1}^{4} \frac{\partial \zeta_{i}}{\partial Z}(p) \frac{\partial \varphi_{1}}{\partial \zeta_{i}}(p), & \sum_{i=1}^{4} \frac{\partial \zeta_{i}}{\partial W}(p) \frac{\partial \varphi_{1}}{\partial \zeta_{i}}(p)
\end{array}\right)
\end{aligned}
$$

On the other hand, by the assumption $(2.29 \mathrm{c})$,

$$
\left|\begin{array}{ll}
\frac{\partial \varphi_{0}}{\partial Z}(p) & \frac{\partial \varphi_{0}}{\partial W}(p) \\
\frac{\partial \varphi_{1}}{\partial Z}(p) & \frac{\partial \varphi_{1}}{\partial W}(p)
\end{array}\right| \neq 0 .
$$

Hence

$$
\left|\begin{array}{ll}
\sum_{i=1}^{4} \frac{\partial \zeta_{i}}{\partial Z}(p) \frac{\partial \varphi_{0}}{\partial \zeta_{i}}(p), & \sum_{i=1}^{4} \frac{\partial \zeta_{i}}{\partial W}(p) \frac{\partial \varphi_{0}}{\partial \zeta_{i}}(p) \\
\sum_{i=1}^{4} \frac{\partial \zeta_{i}}{\partial Z}(p) \frac{\partial \varphi_{1}}{\partial \zeta_{i}}(p), \quad \sum_{i=1}^{4} \frac{\partial \zeta_{i}}{\partial W}(p) \frac{\partial \varphi_{1}}{\partial \zeta_{i}}(p)
\end{array}\right| \neq 0 .
$$

By $(2.30)$ and $(2.31)$, we conclude $\left\{\partial\left(f^{*} \varphi_{0}, f^{*} \varphi_{1}\right) / \partial(u, v, t)\right\}(p)$ has the maximal rank. From this it follows that $\widetilde{C_{\infty}}$ is non-singular at $q$. Furthermore, if $[\alpha: \beta] \neq\left[\alpha^{\prime}: \beta^{\prime}\right]$ as a point of $\mathrm{P}^{1}(\mathbf{C})$, then $\alpha \beta^{\prime}-\alpha^{\prime} \beta \neq 0$, so

$$
\frac{\partial\left(f^{*} \varphi_{0}, f^{*} \varphi_{1}\right)}{\partial(u, v, t)}(q) \quad \text { and } \quad \frac{\partial\left(\alpha f^{*} \varphi_{0}+\beta f^{*} \varphi_{1}, \alpha^{\prime} f^{*} \varphi_{0}+\beta^{\prime} f^{*} \varphi_{1}\right)}{\partial(u, v, t)}(q)
$$

have the same rank. Hence $\left\{\partial\left(\alpha f^{*} \varphi_{0}+\beta f^{*} \varphi_{1}, \alpha^{\prime} f^{*} \varphi_{0}+\beta^{\prime} f^{*} \varphi_{1}\right) / \partial(u, v, t)\right\}(q)$ also has the maximal rank. This shows that assertion (iii) holds at $q$ as required. This completes the proof of the lemma.

Let $\sigma: \widehat{X_{1}} \rightarrow X_{1}$ be the blowing-up along $\widetilde{C_{\infty}}:=f^{-1}\left(C_{\infty}\right)$, and $\widehat{\mathcal{L}}:=\bigcup_{\lambda \in P} \widehat{X_{\lambda}}$ the proper inverse of $\widetilde{\mathcal{L}}:=\bigcup_{\lambda \in P} \widetilde{X_{\lambda}}$. Then $\widehat{\mathcal{L}}$ gives a fibering of $\widehat{X_{1}}$ over $P \simeq P^{1}(\mathbf{C})$, which we denote by $\pi: \widehat{X_{1}} \rightarrow P$. Let $S=\left\{\lambda_{1}, \ldots, \lambda_{c}\right\}$ and $\widehat{X}_{1}^{*}=\widehat{X}_{1}-\pi^{-1}(S)$. From the exact Borel-Moore homology sequence determined by the space, the closed subspace, and its complement, it follows that

$$
\chi\left(\widehat{X_{1}}\right)=\chi\left({\widehat{X_{1}}}^{*}\right)+\chi\left(\pi^{-1}(S)\right) .
$$


It is clear that

$$
\chi\left(\pi^{-1}(S)\right)=\sum_{i=1}^{c} \chi\left(\widehat{X_{\lambda_{i}}}\right) .
$$

Since $\widehat{X}_{1}^{*} \rightarrow P-S$ is locally trivial (as a differential fiber space), it follows from the spectral sequence of Leray for this fiber space that

$$
\chi\left({\widehat{X_{1}}}^{*}\right)=\chi\left({\widehat{X_{\lambda}}}\right) \chi(P-S)
$$

where $\widehat{X_{\lambda}}$ denote a generic fiber of $\widehat{X}_{1}^{*} \rightarrow P-S$. By the same reason as before, we have

$$
\chi(P)=\chi(P-S)+c .
$$

Comparing (2.32), (2.33), (2.34) and (2.35), we have

$$
\chi\left(\widehat{X_{1}}\right)=\chi\left(P^{1}(\mathbf{C})\right) \chi\left(\widehat{X_{\lambda}}\right)+\sum_{j=1}^{c}\left(\chi\left(\widehat{X_{\lambda_{j}}}\right)-\chi\left(\widehat{X_{\lambda}}\right)\right)=2 \chi\left(\widehat{X_{\lambda}}\right)-c .
$$

The second equality above follows from the fact that a topological 2-cycle vanishes when $\lambda \rightarrow \lambda_{j}$ for $j=1, \ldots, c$. We put $\widehat{E}:=\sigma^{-1}\left(\widetilde{C_{\infty}}\right)$. Then, since $\widehat{X_{1}} \backslash \widehat{E} \simeq X_{1} \backslash \widetilde{C_{\infty}}$,

$$
\chi\left(\widehat{X_{1}}\right)-\chi\left(X_{1}\right)=\chi(\widehat{E})-\chi\left(\widetilde{C_{\infty}}\right)=\chi\left(P^{1}(\mathbf{C})\right) \chi\left(\widetilde{C_{\infty}}\right)-\chi\left(\widetilde{C_{\infty}}\right)=\chi\left(\widetilde{C_{\infty}}\right) .
$$

Hence,

$$
\chi\left(X_{1}\right)=\chi\left(\widehat{X_{1}}\right)-\chi\left(\widetilde{C_{\infty}}\right)=2 \chi\left(\widehat{X_{\lambda}}\right)-\chi\left(\widetilde{C_{\infty}}\right)-c=2 \chi\left(\widetilde{X_{\lambda}}\right)-\chi\left(\widetilde{C_{\infty}}\right)-c .
$$

Since $C_{\infty}$ is a curve whose degree is equal to $n$ with $m$ ordinary double points in $P_{\infty} \simeq P^{2}(\mathbf{C})$, the genus $g\left(\widetilde{C_{\infty}}\right)$ is given by

$$
g\left(\widetilde{C_{\infty}}\right)=\frac{1}{2}(n-1)(n-2)-m
$$

Hence,

$$
\chi\left(\widetilde{C_{\infty}}\right)=2-2 g\left(\widetilde{C_{\infty}}\right)=2-(n-1)(n-2)+2 m .
$$

Note that $X_{\lambda}$ is a surface with ordinary singularities in $H_{\lambda} \simeq P^{3}(\mathbf{C})$ of degree $n$, whose numerical characteristics related to its singularities are as follows:

- the degree of its double curve $D_{\lambda}=m$,

- \#\{triple points of $\left.X_{\lambda}\right\}=t$,

- $\#\left\{\right.$ cuspidal points of $\left.X_{\lambda}\right\}=\gamma$.

Therefore, by the classical formula,

$$
\chi\left(\widetilde{X_{\lambda}}\right)=n\left(n^{2}-4 n+6\right)-(3 n-8) m+3 t-2 \gamma .
$$

By (2.36), (2.37) and (2.38), we have the following:

Proposition 2.3.

$$
\begin{array}{r}
\chi\left(X_{1}\right)=2 n\left(n^{2}-4 n+6\right)-2(3 n-8) m+6 t-4 \gamma-2+(n-1)(n-2)-2 m-c \\
=n\left(2 n^{2}-7 n+9\right)-2(3 n-7) m+6 t-4 \gamma-c .
\end{array}
$$


3. The computation of the class of an algebraic threefold with ordinary singularities in $P^{4}(\mathbf{C})$. Throughout this section we denote a rational equivalence class of an algebraic cycle, say $\alpha$, by $[\alpha]$, and denote the intersection class of two algebraic cycle classes, say $[\alpha]$ and $[\beta]$, by $\alpha \cdot \beta$. We refer to the following theorem from [5].

Theorem 3.1 ([5], Theorem (2.3)). Let $X^{n}$ be a hypersurface of degree $d$ in $P^{n+1}$. Then its $k$-th polar class is given by

$$
\left[M_{k}\right]=\left[(d-1) c_{1}(L)\right]^{k} \cap[X]-\sum_{i=0}^{k-1}\left(\begin{array}{c}
k \\
i
\end{array}\right)\left[(d-1) c_{1}(L)\right]^{i} \cap s_{n-k+i}(J, X) \quad(0 \leq k \leq n)
$$

where $L=\mathcal{O}_{P^{n}}(1)$ and $s(J, X)=\sum_{k=0}^{n} s_{k}(J, X),\left(s_{k}(J, X) \in A_{k}(J)\right)$ denotes the Segre class of the singular subscheme $J$ of $X$.

In what follows, using the theorem above, we shall compute the class $c$ of an algebraic threefold with ordinary singularities in the complex projective 4-space $P^{4}(\mathbf{C})$ for the case where the threefold is free from quadruple points. First we fix the notation as follows:

$Y=P^{4}(\mathbf{C}): \quad$ the complex projective 4 -space,

$\bar{X}: \quad$ an algebraic threefold with ordinary singularities in $Y$, which is free from quadruple points,

$\bar{J}: \quad$ the singular subscheme of $\bar{X}$ defined by the Jacobian ideal of $\bar{X}$,

$\bar{D}: \quad$ the singular locus of $\bar{X}$,

$\bar{T}: \quad$ the triple point locus of $\bar{X}$, which is equal to the singular locus of $\bar{D}$,

$\bar{C}: \quad$ the cuspidal point locus of $\bar{X}$, precisely, its closure, since we always consider $\bar{C}$ contains the stationary points,

$\sum \bar{s}: \quad$ the stationary point locus of $\bar{X}$,

$n_{\bar{X}}: X \rightarrow \bar{X}$ : the normalization of $\bar{X}$,

$f: X \rightarrow Y:$ the composite of the normalization map $n_{\bar{X}}$ and the inclusion $\iota: \bar{X} \hookrightarrow Y$, $J: \quad$ the scheme-theoretic inverse of $\bar{J}$ by $f$,

$D, T, C: \quad$ the inverse images of $\bar{D}, \bar{T}, \bar{C}$ by $f$, respectively,

$\sum s=T \cap C:$ the intersection of $T$ and $C$.

Note that $\bar{T}$ and $\bar{C}$ are non-singular curves, intersecting transversely at $\sum \bar{s}$, and that the normalization $X$ of $\bar{X}$ is also non-singular. Calculating by use of local coordinates, we can easily see the following:

(i) $J$ contains $D$, and the residual scheme (cf. [2], Definition 9.2.1, p. 160) to $D$ in $J$ is the reduced scheme $C$;

(ii) $D$ is a surface with ordinary singularities, free from triple points, whose singular locus is $T$,

(iii) $D$ is the double point locus of the map $f: X \rightarrow Y$, i.e., the closure of the set $\left\{q \in X \mid \# f^{-1}(f(q)) \geq 2\right\}$;

(iv) the map $f_{\mid D}: D \rightarrow \bar{D}$ is generically two to one, simply ramified at $C$;

(v) the map $f_{\mid T}: T \rightarrow \bar{T}$ is generically three to one, simply ramified at $\sum s$.

To compute the Segre class $s(J, X)$, the following proposition is useful. 
Proposition 3.2 ([2], Proposition 9.2, p. 161). Let $D \subset W \subset V$ be closed embeddings of schemes, with $V$ a $k$-dimensional variety, and $D$ a Cartier divisor on $V$. Let $R$ be the residual scheme to $D$ in $W$. Then, for all $m$,

$$
s(W, V)_{m}=s(D, V)_{m}+\sum_{j=0}^{k-m}\left(\begin{array}{c}
k-m \\
j
\end{array}\right)[-D]^{j} \cdot s(R, V)_{m+j}
$$

in $A_{m}(W)$, the $m$-th rational equivalence class group of algebraic cycles on $W$.

In our case, since $D=f^{-1}(\bar{D})$ is a Cartier divisor, its normal cone $C_{D} X$ to $D$ in $X$ is isomorphic to $\mathcal{O}_{X}(D)_{\mid D}$, the restriction to $D$ of the line bundle $\mathcal{O}_{X}(D)$ associated to $D$. Therefore,

$$
\begin{aligned}
s(D, X)= & c\left(\mathcal{O}_{X}(D)_{\mid D}\right)^{-1} \cap[D] \\
& =[D]-c_{1}\left(\mathcal{O}_{X}(D)_{\mid D}\right) \cap[D]+c_{1}\left(\mathcal{O}_{X}(D)_{\mid D}\right)^{2} \cap[D]=[D]-[D]^{2}+[D]^{3} .
\end{aligned}
$$

Since $C$ is non-singular,

$$
c\left(N_{C / X}\right)^{-1} \cap[C]=[C]-c_{1}\left(N_{C / X}\right) \cap[C] .
$$

Hence, applying Proposition 3.2 for $W=J, D=f^{-1}(\bar{D})$ and $R=C$, we have

$$
\left\{\begin{array}{l}
s(J, X)_{2}=[D] \\
s(J, X)_{1}=-[D]^{2}+[C] \\
s(J, X)_{0}=[D]^{3}-c_{1}\left(N_{C / X}\right) \cap[C]-3 D \cdot C
\end{array}\right.
$$

Since $s(\bar{J}, \bar{X})_{2}=f_{*} s(J, X)_{2}$, from the first equality above it follows that

$$
s(\bar{J}, \bar{X})_{2}=2[\bar{D}]
$$

To know $s(\bar{J}, \bar{X})_{1}$, we need to understand $f_{*}[D]^{2}$, the push-forward of $[D]^{2}$ by $f$. For this purpose, we compute $f^{*}[D]^{2}$. To compute this, we consider the following fiber square:

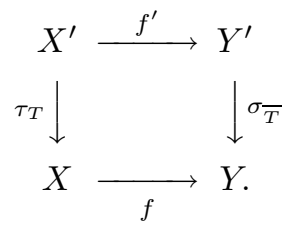

Here

$\sigma_{\bar{T}}: Y^{\prime} \rightarrow Y: \quad$ the blowing-up of $Y$ along the triple point locus $\bar{T}$ of $\bar{X}$,

$\overline{X^{\prime}}: \quad$ the proper inverse image of $\bar{X}$ by $\sigma_{\bar{T}}$,

$X^{\prime}:=X \times_{\bar{X}} \overline{X^{\prime}}$ : the fiber product of $X$ and $\overline{X^{\prime}}$ over $\bar{X}$,

$n_{\overline{X^{\prime}}}: X^{\prime} \rightarrow \overline{X^{\prime}}: \quad$ the projection to the second factor of $X \times_{\bar{X}} \overline{X^{\prime}}$, which is nothing but the normalization of $\overline{X^{\prime}}$,

$f^{\prime}: X^{\prime} \rightarrow Y^{\prime}: \quad$ the composite of the normalization map $n_{\overline{X^{\prime}}}$ and the inclusion $\iota^{\prime}: \overline{X^{\prime}} \hookrightarrow Y^{\prime}$,

$\tau_{T}: X^{\prime} \rightarrow X: \quad$ the projection to the first factor of $X \times \bar{X} \overline{X^{\prime}}$, which is nothing but the blowing-up of $X$ along $T$. 
In what follows, we denote by $\overline{D^{\prime}}, \overline{T^{\prime}}$ and $\overline{C^{\prime}}$ the proper inverse images of $\bar{D}, \bar{T}$ and $\bar{C}$ by $\sigma_{\bar{T}}$, respectively. We consider the following fiber square:

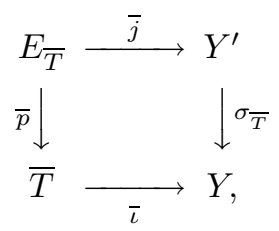

where $E_{\bar{T}}=P\left(N_{\bar{T}} Y\right)$ is the exceptional divisor of the blowing-up $\sigma_{\bar{T}}$, which is a $P^{2}(\mathbf{C})$ bundle on $\bar{T}$, and $\bar{p}: E_{\bar{T}} \rightarrow \bar{T}$ is the projection to the base space of this bundle. We denote by $\mathcal{O}_{N_{\bar{T}} Y}(1)$ the canonical line bundle on $E_{\bar{T}}$. Then the tautological line bundle on $E_{\bar{T}}$ is $\mathcal{O}_{N_{\bar{T}} Y}(-1)$, which is a subbundle of $\bar{p}^{*} N_{\bar{T}} Y$.

LEMMA 3.3. $\sigma_{\bar{T}}^{*}[\bar{D}]$ is expressed as

$$
\sigma_{\bar{T}}^{*}[\bar{D}]=\left[\overline{D^{\prime}}\right]+3 \bar{j}_{*}\left[\xi_{\bar{T}}\right]+\bar{j}_{*} \bar{p}^{*}\left[\alpha_{0}\right]
$$

where $\left[\xi_{\bar{T}}\right]=c_{1}\left(\mathcal{O}_{N_{\bar{T}} Y}(1)\right) \cap\left[E_{\bar{T}}\right]$ and $\left[\alpha_{0}\right]$ an algebraic 0 -cycle class on $\bar{T}$.

Proof. By the blow-up formula ([2], Theorem 6.7, p. 116),

$$
\sigma_{\bar{T}}^{*}[\bar{D}]=\left[\overline{D^{\prime}}\right]+\bar{j}_{*}\left\{c(E) \cap \bar{p}^{*} s(\bar{T}, \bar{D})\right\}_{2}
$$

where $E=\bar{p}^{*} N_{\bar{T}} Y / N_{E_{\bar{T}}} Y^{\prime}=\bar{p}^{*} N_{\bar{T}} Y / \mathcal{O}_{N_{\bar{T}} Y}(-1)$. Since

$$
c_{1}(E)=\bar{p}^{*} c_{1}\left(N_{\bar{T}} Y\right)-c_{1}\left(\mathcal{O}_{N_{\bar{T}} Y}(-1)\right)=\bar{p}^{*} c_{1}\left(N_{\bar{T}} Y\right)+c_{1}\left(\mathcal{O}_{N_{\bar{T}} Y}(1)\right),
$$

we have

$$
\begin{aligned}
\{c(E) & \cap s(\bar{T}, \bar{D})\}_{2}=\bar{p}^{*} s_{0}(\bar{T}, \bar{D})+c_{1}(E) \cap \bar{p}^{*} s_{1}(\bar{T}, \bar{D}) \\
& =\bar{p}^{*}\left\{s_{0}(\bar{T}, \bar{D})+c_{1}\left(N_{\bar{T}} Y\right) \cap s_{1}(\bar{T}, \bar{D})\right\}+c_{1}\left(\mathcal{O}_{N_{\bar{T}} Y}(1)\right) \cap \bar{p}^{*} s_{1}(\bar{T}, \bar{D})
\end{aligned}
$$

To compute $s(\bar{T}, \bar{D})$, we consider the normalization map $n_{\bar{D}}: \bar{D}^{*} \rightarrow \bar{D}$. Since $\bar{D}^{*}$ is non-singular, if we put $\bar{T}^{*}:=n_{\bar{D}}^{-1}(\bar{T})$, then

$$
s\left(\bar{T}^{*}, \bar{D}^{*}\right)=c\left(N_{\bar{T}^{*}} \bar{D}^{*}\right)^{-1} \cap\left[\bar{T}^{*}\right]=\left(1-c_{1}\left(N_{\bar{T}^{*}} \bar{D}^{*}\right)\right) \cap\left[\bar{T}^{*}\right]=\left[\bar{T}^{*}\right]-\bar{T}^{*} \cdot \bar{T}^{*} .
$$

Therefore,

$$
s(\bar{T}, \bar{D})=n_{\bar{D}_{*}} s\left(\bar{T}^{*}, \bar{D}^{*}\right)=3[\bar{T}]-n_{\bar{D}_{*}}\left(\bar{T}^{*} \cdot \bar{T}^{*}\right)
$$

and so,

$$
\left\{\begin{array}{l}
s_{0}(\bar{T}, \bar{D})=-n_{\bar{D}_{*}}\left(\bar{T}^{*} \cdot \bar{T}^{*}\right) \\
s_{1}(\bar{T}, \bar{D})=3[\bar{T}] .
\end{array}\right.
$$

By (3.7) and (3.8), if we put $\left[\alpha_{0}\right]:=-n_{\bar{D}_{*}}\left(\bar{T}^{*} \cdot \bar{T}^{*}\right)+3 c_{1}\left(N_{\bar{T}} Y\right) \cap[\bar{T}]$,

$$
\{c(E) \cap s(\bar{T}, \bar{D})\}_{2}=\bar{p}^{*}\left[\alpha_{0}\right]+3\left[\xi_{\bar{T}}\right] .
$$

Consequently, by (3.6), we have the equality in (3.5).

Proposition 3.4.

$$
[D]^{2}=f^{*}[\bar{X}] \cdot D-f^{*}[\bar{D}]+[T]-[C] .
$$


Proof. To know $[D]^{2}$, we compute $f^{*}[\bar{D}]$. For this purpose, we use the diagram in (3.3). Since $\tau_{T}: X^{\prime} \rightarrow X$ is a blowing-up, we have $\tau_{T_{*}} \tau_{T}^{*} \alpha=\alpha$ for any algebraic cycle $\alpha \in A_{*}(X)$. Hence,

$$
\tau_{T_{*}} f^{\prime *} \sigma_{\bar{T}}^{*}[\bar{D}]=\tau_{T_{*}} \tau_{T}^{*} f^{*}[\bar{D}]=f^{*}[\bar{D}] .
$$

Since $\overline{D^{\prime}}$ is regularly embedded in $Y^{\prime}$, i.e., $C_{\overline{D^{\prime}}} Y^{\prime} \simeq N_{\overline{D^{\prime}}} Y^{\prime}$, while $\bar{D}$ is not, we can apply the excess intersection formula ([2], Theorem 6.3, p. 102) to $\overline{D^{\prime}}$. Then, denoting the tangent bundle of a non-singular algebraic variety, say $Z$, by $\mathcal{T}_{Z}$ we have

$$
\begin{aligned}
f^{\prime *}\left[\overline{D^{\prime}}\right] & =c_{1}\left(f^{\prime *} N_{\overline{D^{\prime}}} Y^{\prime} / N_{D^{\prime}} X^{\prime}\right) \cap\left[D^{\prime}\right] \\
& =\left\{c_{1}\left(f^{\prime *} \mathcal{T}_{Y^{\prime}}\right)-c_{1}\left(f^{\prime *} \mathcal{T}_{\overline{D^{\prime}}}\right)-c_{1}\left(\mathcal{T}_{X^{\prime}}\right)+c_{1}\left(\mathcal{T}_{D^{\prime}}\right)\right\} \cap\left[D^{\prime}\right] \\
& =\left\{c_{1}\left(f^{\prime *} \mathcal{T}_{Y^{\prime}}\right)-c_{1}\left(\mathcal{T}_{X^{\prime}}\right)\right\} \cap\left[D^{\prime}\right]-C^{\prime},
\end{aligned}
$$

where the last equality follows from the ramification formula ([2], Example 3.2.20, p. 62). On the other hand, by the double point formula ([2], Theorem 9.3, p. 166),

$$
\left[D^{\prime}\right]=f^{\prime *}\left[\overline{X^{\prime}}\right]-\left\{c_{1}\left(f^{\prime *} \mathcal{T}_{Y^{\prime}}\right)-c_{1}\left(\mathcal{T}_{X^{\prime}}\right)\right\} \cap\left[X^{\prime}\right]
$$

By (3.11) and (3.12), we have

$$
f^{\prime *}\left[\overline{D^{\prime}}\right]=f^{\prime *}\left[\overline{X^{\prime}}\right] \cdot D^{\prime}-\left[D^{\prime}\right]^{2}-C^{\prime} .
$$

Next, in view of Lemma 3.3, we compute $f^{\prime *}\left(3 \bar{j}_{*}\left[\xi_{\bar{T}}\right]+\bar{j}_{*} \bar{p}^{*}\left[\alpha_{0}\right]\right)$. For this purpose, we consider the following fiber square:

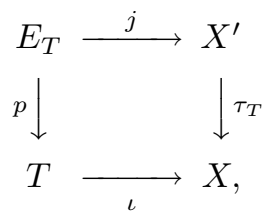

where $E_{T}=P\left(N_{T} X\right)$ is the exceptional divisor of the blowing-up $\tau_{T}$, which is a $P^{1}(\mathbf{C})$ bundle on $T$, and $p: E_{T} \rightarrow T$ is the projection to the base space of this bundle. There is a set of morphisms from the diagram in (3.14) to the one in (3.4) induced by those in the diagram in (3.3). We denote by $g$ and $g^{\prime}$ the restriction of $f: X \rightarrow Y$ to $T$ and that of $f^{\prime}: X^{\prime} \rightarrow Y^{\prime}$ to $E_{T}$, respectively. Note that the morphism $g^{\prime}: E_{T} \rightarrow E_{\bar{T}}$ maps each fiber of $p: E_{T} \rightarrow T$ to that of $\bar{p}: E_{\bar{T}} \rightarrow \bar{T}$, and so $g^{\prime a} s t\left[\xi_{\bar{T}}\right]=\left[\xi_{T}\right]$ where $\xi_{T}=c_{1}\left(\mathcal{O}_{N_{T} X}(1)\right) \cap\left[E_{T}\right]$. Since $f^{\prime}: X^{\prime} \rightarrow Y^{\prime}$ and $g^{\prime}: E_{T} \rightarrow E_{\bar{T}}$ are local complete intersection morphisms of the same codimension, we can apply Proposition 6.6(c) from [2], p. 113 , to the fiber square

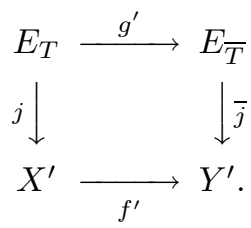

Then $f^{\prime *} \bar{j}_{*}\left[\xi_{\bar{T}}\right]=j_{*} g^{\prime *}\left[\xi_{\bar{T}}\right]=j_{*}\left[\xi_{T}\right]$ and $f^{\prime *} \bar{j}_{*} \bar{p}^{*}\left[\alpha_{0}\right]=j_{*} g^{\prime a} s t \bar{p}^{*}\left[\alpha_{0}\right]=j_{*} p^{*} g^{*}\left[\alpha_{0}\right]$. Therefore, we have

$$
f^{\prime *}\left(3 \bar{j}_{*}\left[\xi_{\bar{T}}\right]+\bar{j}_{*} \bar{p}^{*}\left[\alpha_{0}\right]\right)=3 j_{*}\left[\xi_{\bar{T}}\right]+\bar{j}_{*} \bar{p}^{*} g^{*}\left[\alpha_{0}\right]
$$


By (3.5), (3.13) and (3.16), we have

$$
f^{\prime *} \sigma_{\bar{T}}^{*}[\bar{D}]=f^{\prime *}\left[\overline{X^{\prime}}\right] \cdot D^{\prime}-\left[D^{\prime}\right]^{2}-C^{\prime}+3 j_{*}\left[\xi_{\bar{T}}\right]+j_{*} \bar{p}^{*} g^{*}\left[\alpha_{0}\right] .
$$

Since $\tau_{T_{*}}\left[C^{\prime}\right]=[C], \tau_{T_{*}} j_{*}\left[\xi_{\bar{T}}\right]=T$ and $\tau_{T_{*}} j_{*} \bar{p}^{*} g^{*}\left[\alpha_{0}\right]=0$, by the equality above and (3.10),

$$
f^{*}[\bar{D}]=\tau_{T_{*}} f^{\prime *} \sigma_{\bar{T}}^{*}[\bar{D}]=\tau_{T_{*}}\left(f^{\prime *}\left[\overline{X^{\prime}}\right] \cdot D^{\prime}\right)-\tau_{T_{*}}\left[D^{\prime}\right]^{2}-[C]+3[T] .
$$

Since $\tau_{T}^{*}[D]=\left[D^{\prime}\right]+2\left[E_{T}\right]$,

$$
\tau_{T_{*}}\left(f^{\prime *}\left[\overline{X^{\prime}}\right] \cdot D^{\prime}\right)=\tau_{T_{*}}\left(f^{\prime *}\left[\overline{X^{\prime}}\right] \cdot \tau_{T}^{*}[D]-2 f^{\prime *}\left[\overline{X^{\prime}}\right] \cdot E_{T}\right) .
$$

On the other hand, since $\sigma_{\bar{T}}^{*}[\bar{X}]=\left[\overline{X^{\prime}}\right]+3\left[E_{\bar{T}}\right]$,

$$
f^{\prime *}\left[\overline{X^{\prime}}\right]=f^{\prime *} \sigma_{T}^{*}[\bar{X}]-3\left[E_{T}\right] .
$$

Hence, by the projection formula,

$$
\tau_{T_{*}}\left(f^{\prime *}\left[\overline{X^{\prime}}\right] \cdot \tau_{T}^{*}[D]\right)=\tau_{T_{*}}\left(f^{\prime *}\left[\overline{X^{\prime}}\right]\right) \cdot D=\tau_{T_{*}}\left(f^{\prime *} \sigma_{\bar{T}}^{*}\left[\overline{X^{\prime}}\right]\right) \cdot D=f^{*}[\bar{X}] \cdot D
$$

and

$$
\begin{aligned}
& \tau_{T_{*}}\left(f^{\prime *}\left[\overline{X^{\prime}}\right] \cdot E_{T}\right)=\tau_{T_{*}}\left(f^{\prime *} \sigma_{\bar{T}}^{*}[\bar{X}] \cdot E_{T}-3\left[E_{T}\right]^{2}\right) \\
& \quad=\tau_{T_{*}}\left(\tau_{T}^{*} f^{*}[\bar{X}] \cdot E_{T}\right)+3 \tau_{T_{*}} j_{*}\left[\xi_{T}\right]=f^{*}[\bar{X}] \cdot \tau_{T_{*}}\left[E_{T}\right]+3 i_{*}[T]=3[T]
\end{aligned}
$$

Therefore, by (3.18), (3.19) and (3.20),

$$
\tau_{T_{*}}\left(f^{\prime *}\left[\overline{X^{\prime}}\right] \cdot D^{\prime}\right)=f^{*}[\bar{X}] \cdot D-6[T] .
$$

Furthermore, we have

$$
\begin{aligned}
\tau_{T_{*}}\left[D^{\prime}\right]^{2} & =\tau_{T_{*}}\left(\left(\tau_{T}^{*}[D]-2\left[E_{T}\right]\right)^{2}\right) \\
& =\tau_{T_{*}}\left(\left(\tau_{T}^{*}[D]\right)^{2}-4 \tau_{T}^{*}[D] \cdot\left[E_{T}\right]+4\left[E_{T}\right]^{2}\right) \\
& =\tau_{T_{*}}\left(\tau_{T}^{*}[D]\right) \cdot D-4 D \cdot \tau_{T_{*}}\left[E_{T}\right]-4 \tau_{T_{*}} j_{*}\left[\xi_{T}\right]=[D]^{2}-4[T] .
\end{aligned}
$$

Consequently, by (3.17), (3.21) and (3.22),

$$
\begin{aligned}
f^{*}[\bar{D}]=f^{*}[\bar{X}] \cdot D-6[T]-[D]^{2}+4[T]-[C]+3[T] & =f^{*}[\bar{X}] \cdot D-[D]^{2}-[C]+[T],
\end{aligned}
$$

from which equality (3.9) follows.

Since $f_{*}[X]=[\bar{X}], f_{*}[D]=2[\bar{D}], f_{*}[T]=3[\bar{T}]$ and $f_{*}[C]=[\bar{C}]$, by Proposition 3.4, we have the following:

Corollary 3.5.

$$
f_{*}[D]^{2}=\bar{X} \cdot \bar{D}+3[\bar{T}]-[\bar{C}]
$$

By Proposition 3.4 and the second equality in (3.1),

$$
s(J, X)_{1}=-f^{*}[\bar{X}] \cdot D+f^{*}[\bar{D}]-[T]+2[C]
$$

and so, by the projection formula

$$
s(\bar{J}, \bar{X})_{1}=-\bar{X} \cdot \bar{D}-3[\bar{T}]+2[\bar{C}] .
$$

Now we compute $s(\bar{J}, \bar{X})_{0}$. By Proposition 3.4,

$$
[D]^{3}=f^{*}[\bar{X}] \cdot[D]^{2}-f^{*}[\bar{D}] \cdot D+D \cdot T-D \cdot C .
$$


Hence, by the third equality in (3.1),

$$
s(J, X)_{0}=f^{*}[\bar{X}] \cdot[D]^{2}-f^{*}[\bar{D}] \cdot D+D \cdot T-4 D \cdot C-c_{1}\left(N_{C} X\right) \cap[C] .
$$

Since $\bar{T}$ and $\bar{C}$ are regularly embedded in $Y$, we can apply the excess intersection formula to them. Then,

$$
\begin{aligned}
f^{*}[\bar{T}] & =c_{1}\left(f^{*} N_{\bar{T}} Y / N_{T} X\right) \cap[T] \\
& =\left\{c_{1}\left(f^{*} \mathcal{T}_{Y}\right)-c_{1}\left(f^{*} \mathcal{T}_{\bar{T}}\right)-c_{1}\left(\mathcal{T}_{X}\right)+c_{1}\left(\mathcal{T}_{T}\right)\right\} \cap[T] \\
& =\left\{c_{1}\left(f^{*} \mathcal{T}_{Y}\right)-c_{1}\left(\mathcal{T}_{X}\right)\right\} \cap[T]-\left[\sum s\right] \\
& =f^{*}[\bar{X}] \cdot T-D \cdot T-\left[\sum s\right]
\end{aligned}
$$

where the last but one step follows from the ramification formula for $g: T \rightarrow \bar{T}$ and the last step from the double point formula for $f: X \rightarrow Y$. Similarly, since $\bar{C} \simeq C$, we have

$$
f^{*}[\bar{C}]=f^{*}[\bar{X}] \cdot C-D \cdot C .
$$

Therefore we have

$$
\left\{\begin{array}{l}
D \cdot T=f^{*}[\bar{X}] \cdot T-f^{*}[\bar{T}]-\left[\sum s\right] \\
D \cdot C=f^{*}[\bar{X}] \cdot C-f^{*}[\bar{C}]
\end{array}\right.
$$

By the adjunction formula, the double point formula for $f: X \rightarrow Y$ and the second equality in (3.26),

$$
\begin{aligned}
c_{1}\left(N_{C} X\right) & \cap[C]=-K_{X} \cdot C+\left[k_{C}\right] \\
= & \left(-f^{*}\left[\bar{X}+K_{Y}\right]+D\right) \cdot C+\left[k_{C}\right]=-f^{*}\left[K_{Y}\right] \cdot C-f^{*}[\bar{C}]+\left[k_{C}\right],
\end{aligned}
$$

where $K_{Y}, K_{X}$ and $k_{C}$ are the canonical divisors of $Y, X$ and $C$, respectively. Substituting (3.26) and (3.27) into (3.25), we have

$$
\begin{aligned}
s(J, X)_{0}=f^{*}[\bar{X}] \cdot[D]^{2}-f^{*}[\bar{D}] \cdot D+f^{*}[\bar{X}] \cdot T-f^{*}[\bar{T}]-\left[\sum s\right] \\
\\
-4 f^{*}[\bar{X}] \cdot C+4 f^{*}[\bar{C}]+f^{*}\left[K_{Y}\right] \cdot C+f^{*}[\bar{C}]-\left[k_{C}\right] .
\end{aligned}
$$

Consequently, using Corollary 3.5 and the fact that $f_{*}[X]=[\bar{X}], f_{*}[D]=2[\bar{D}]$, $f_{*}[T]=3[\bar{T}], f_{*}\left[\sum s\right]=\left[\sum \bar{s}\right]$ and $\bar{C} \simeq C$, we have,

$$
s(\bar{J}, \bar{X})_{0}=[\bar{X}]^{2} \cdot \bar{D}-2[\bar{D}]^{2}+5 \bar{X} \cdot \bar{T}+K_{Y} \cdot \bar{C}-\left[k_{\bar{C}}\right]-\left[\sum \bar{s}\right] .
$$

We collect the results obtained till now in the following proposition:

Proposition 3.6. The Segre classes of the singular subscheme $\bar{J}$, defined by the Jacobian ideal, of an algebraic threefold $\bar{X}$ with ordinary singularities in the four-dimensional projective space $Y=P^{4}(\mathbf{C})$ are given as follows, if $\bar{X}$ is free from quadruple points:

$$
\left\{\begin{array}{l}
s(\bar{J}, \bar{X})_{2}=2[\bar{D}] \\
s(\bar{J}, \bar{X})_{1}=-\bar{X} \cdot \bar{D}-3 \bar{T}+2 \bar{C} \\
s(\bar{J}, \bar{X})_{0}=[\bar{X}]^{2} \cdot \bar{D}-2[\bar{D}]^{2}+5 \bar{X} \cdot \bar{T}+K_{Y} \cdot \bar{C}-\left[k_{\bar{C}}\right]-\left[\sum \bar{s}\right]
\end{array}\right.
$$

Here $\bar{D}, \bar{T}, \bar{C}$ and $\sum \bar{s}$ are the singular locus, triple point locus, cuspidal point locus and stationary point locus of $\bar{X}$, respectively. $K_{Y}$ is the canonical divisor of the projective 4-space $Y$, and $k_{\bar{C}}$ that of $\bar{C}$. 
4. The Euler number of the normalization of an algebraic threefold with ordinary singularities. By Theorem 3.1, the top polar class $\left[M_{3}\right]$ of $\bar{X}$ is given by

$$
\left[M_{3}\right]=(n-1)^{3} h^{3}-3(n-1)^{2} h^{2} \cap s_{2}-3(n-1) h \cap s_{1}-s_{0},
$$

where $h$ denotes the hyperplane section class and $s_{i} i$-th Segre class $s(\bar{J}, \bar{X})_{i}(0 \leq i \leq 2)$ and $n=\operatorname{deg} \bar{X}$, the degree of $\bar{X}$ in $Y$. We put

$$
m=\operatorname{deg} \bar{D}, t=\operatorname{deg} \bar{T}, \gamma=\operatorname{deg} \bar{C} \text { and } \# \sum \bar{s}=\text { the cardinal number of } \sum \bar{s} .
$$

Then, by Proposition 3.6,

$$
\left\{\begin{array}{l}
\operatorname{deg} s_{2}=2 m \\
\operatorname{deg} s_{1}=-n m+2 \gamma-3 t \\
\operatorname{deg} s_{0}=n^{2} m-2 m^{2}+5 n t-5 \gamma-\# \sum \bar{s}-\operatorname{deg} k_{\bar{C}} .
\end{array}\right.
$$

Consequently, the class $c$ of $\bar{X}$ is given by

$$
\begin{aligned}
c & =\operatorname{deg}\left[M_{3}\right]=(n-1)^{3} \operatorname{deg} \bar{X}-3(n-1)^{2} \operatorname{deg} s_{2}-3(n-1) \operatorname{deg} s_{1}-\operatorname{deg} s_{0} \\
& =(n-1)^{3} n-\left(4 n^{2}-9 n-2 m+6\right) m+(4 n-9) t-(6 n-11) \gamma+\# \sum \bar{s}+\operatorname{deg} k_{\bar{C}} .
\end{aligned}
$$

By this formula together with Proposition 2.3, we have the following:

THEOREM 4.1. The Euler number $\chi(X)$ of the non-singular normalization $X$ of an algebraic threefold $\bar{X}$ with ordinary singularities in $P^{4}(\mathbf{C})$ which is free from quadruple points is given by

$$
\begin{aligned}
\chi(X)=-n\left(n^{3}-5 n^{2}+10 n-10\right)+\left(4 n^{2}-15 n-2 m+20\right) m & -(4 n-15) t \\
& +(6 n-15) \gamma-\# \sum \bar{s}-\operatorname{deg} k_{\bar{C}} .
\end{aligned}
$$

Here $n=\operatorname{deg} \bar{X}, m=\operatorname{deg} \bar{D}, t=\operatorname{deg} \bar{T}$ and $\gamma=\operatorname{deg} \bar{C}$ are the degrees of $\bar{X}$, the singular locus, the triple point locus and the cuspidal point locus, respectively. $\# \sum \bar{s}$ is the cardinal number of the stationary point locus $\sum \bar{s}$, and $\operatorname{deg} k_{\bar{C}}$ the degree of the canonical divisor of the cuspidal point locus $\bar{C}$.

Acknowledgements. In the first version of this article, the author made a serious mistake with respect to the signature of the class number $c$ of an algebraic threefold with ordinary singularities in $P^{4}(\mathbf{C})$ in the formula $(2.36)$. The author is very grateful to the referee for his suggestion which makes the author become aware of the mistake.

\section{References}

[1] F. Enriques, Le Superficie Algebriche, Nicola Zanichelli, Bologna, 1949.

[2] W. Fulton, Intersection Theory, Ergeb. Math. Grenzgeb. (3) 2, Springer, Berlin, 1984.

[3] J. Harris, Algebraic Geometry. A First Course, Grad. Texts in Math. 133, Springer, New York, 1992.

[4] A. Nobile, Some properties of the Nash blowing-up, Pacific J. Math. 60 (1975), 297-305.

[5] R. Piene, Polar classes of singular varieties, Ann. Sci. École Norm. Sup. (4) 11 (1978), 247-276.

[6] L. Roth, Algebraic Threefold, Springer, Berlin, 1955.

[7] F. L. Zak, Tangents and Secants of Algebraic Varieties, Transl. Math. Monogr. 127, Amer. Math. Soc., Providence, 1993. 